\title{
Un servicio británico de intervención precoz en psicosis
}

RESUMEN: Se describen las estrategias de "seguimiento asertivo", tratamiento a domicilio, el papel de la enfermería de salud mental comunitaria y el esfuerzo de desestigmatización como pilares de un programa innovador de intervención precoz en psicosis desarrollado en Birmingham (Reino Unido).

PALABRAS CLAVE: Intervención precoz, psicosis, enfermería psiquiátrica comunitaria.
SUMMARY: The strategies of assertive outreach, home treatment, the communitary mental health nurse's role and the nonstigmatising efforts are described as the basis of an innovative programme in early intervention in psychosis developed in Birmingham (U.K.).

KEY WORDS: Early intervention, psychosis, communitary psychiatric nurse.

\section{Introducción}

La atención de salud mental a las patologías psiquiátricas severas , como es la psicosis, ha planteado y plantea una problemática propia dentro de la sanidad que requiere una formación específica y modelos a seguir por parte de los profesionales que atendemos dichas patologías.

En el caso de la psicosis existe un modelo interesante en el Centro de Intervención Precoz en Psicosis del North Birmingham Mental Health Trust en el que he realizado como residente de psiquiatría mi rotación de elección libre a la que da acceso el programa de formación de residentes de esta especialidad del Hospital Gregorio Marañón de Madrid. Durante mi estancia he podido ser testigo privilegiado del trabajo que allí se realiza y participar activamente, en horario de 9 a 17 horas, en actividades clínicas como evaluación de pacientes nuevos, discusión de los planes de tratamiento, visitas a domicilio de carácter médico, psicoterapéutico o psicosocial, incluyéndome como una persona más del equipo y sin limitarme al rol del psiquiatra para poder observar y participar del trabajo de cada uno de los miembros del equipo y así traer una visión global del modelo.

Considero la experiencia de gran valor para utilizarla en los aspectos difíciles del trabajo con psicóticos como son, sobre todo, la dificultad de vincularlos al tratamiento y de evitar el aislamiento y deterioro personal y social que, en los primeros años de enfermedad, truncan la vida de estos sujetos justo en el momento en que comienzan su vida laboral, el establecimiento de su independencia, de lazos sociales, de pareja, etc. 
A continuación haré una descripción de los aspectos más destacables y rasgos diferenciales de la atención que allí se brinda y que creo de interés debido a la novedad que supone para la orientación del trabajo en relación con la actividad de nuestra red de salud mental comunitaria.

El Servicio de Intervención Precoz es un centro de atención de salud mental comunitaria específico para el tratamiento de la psicosis en estadíos precoces. Atiende dos distritos (unos $\mathbf{8 5 . 0 0 0}$ habitantes) que albergan una población de nivel socioeconómico bajo, con gran proporción de inmigrantes (principalmente afrocaribeños -jamaicanos- y surasiáticos -indios y pakistaníes-).

Este servicio se estableció en el año 1995 y es el único, exceptuando el Centro de Prevención e Intervención en Psicosis Temprana (EPPIC) dirigido por el Dr. McGorry en Australia (1), que ha desarrollado un programa específico dentro del Servicio Nacional de Salud para esta patología, siendo, pues, pionero en este campo y por ello interesante desde el punto de vista de las posibilidades que ofrece tanto de asistencia como de formación e investigación.

La organización de la salud mental comunitaria en esta zona del Reino Unido es, por sí misma digna de reseñar. Anteriormente a 1995, cada distrito del norte de Birmingham disponía de un solo equipo de salud mental comunitaria a la manera de los nuestros : estos equipos atendían pacientes mentales de todas las edades, independientemente del diagnóstico o severidad de la enfermedad.

En 1995 el Trust de Salud Mental del Norte de Birmingham decidió cambiar su estructura: los equipos de salud mental se disgregaron en servicios más pequeños cada uno de los cuales habría de tener un papel diferenciado y trabajar con un grupo específico de pacientes .

Actualmente, y a consecuencia de dichos cambios, existen cinco servicios componentes de los Equipos de Salud Mental del norte de Birmingham:

-Interconsulta con atención primaria.

-Equipo de "seguimiento asertivo" (Assertive Outreach Team).

-Cuidados continuados.

-Tratamiento a domicilio.

-Intervención Precoz en Psicosis.

El concepto de "seguimiento asertivo", como he creido adecuado traducir assertive outreach, consiste en equipos que proveen apoyo comunitario a pacientes con enfermedad mental severa que tienen dificultades para acceder o aceptar los servicios. Esta concepción de la asistencia sanitaria está pues orientada a hacer accesibles e incluso atractivos los servicios de salud mental para unos pacientes de los que, por su particular patología, 
INFORMES

no se puede esperar que busquen ayuda o acepten los tratamientos facilmente : pacientes sin conciencia de enfermedad, desvinculados de la red social y sanitaria por su situación económica o por la naturaleza de su patología ( varias patologías concurrentes incluyendo alcoholismo, otras drogadicciones, cuadros psicóticos, etc...).

El equipo de Tratamiento a Domicilio(Home Treatment) trabaja con la consigna de evitar al máximo el ingreso de los pacientes mentales en crisis (suicidas, reagudizaciones psicóticas, afectivas...), es un apoyo médico, psicoterapéutico y de enfermería brindado en la propia casa del paciente a través de visitas hasta tres o cuatro veces al día para evaluación, apoyo, medicación... que ahorra internamientos costosos e indeseados en muchas ocasiones.

El equipo de intervención precoz está específicamente concebido para trabajar con pacientes jóvenes que experimentan su primer episodio psicótico y provee ayuda especializada en el contexto de un modelo de "seguimiento asertivo".

\section{El programa de psicosis precoz}

Está basado en la prevención secundaria sobre el reconocimiento de que los primeros años tras el desencadenamiento de una psicosis constituyen un periodo crítico que afecta al pronóstico, siendo los procesos deteriorantes mâs activos en este periodo (2) y alcanzándose después un efecto plateau y un mayor grado de irreversibilidad biológica y psicosocial de los estragos de la psicosis (3).

La intervención precoz reconoce por un lado, la necesidad de una identificación y tratamiento precoces y por otro la heterogeneidad de la psicosis en sus presentaciones y etiología, siendo, por tanto, imprescindibles, intervenciones individualizadas (según la edad y características étnicas y culturales) (4) (5), desarrolladas en un entorno no estigmatizante.

Si pretendemos que los pacientes jóvenes acudan a dispositivos de atención de salud mental y más aún, tratándose de pacientes con precaria o nula conciencia de enfermedad, hemos de crear servicios atractivos para este tipo de usuarios. Esto lo han logrado ofreciendo, por un lado, un ambiente alejado de la tradicional imagen del centro de atención psiquiátrica - que para tantas personas supone rechazo por lo que conlleva de estigmatización del que allí acude- y por otro, ayuda en aspectos económicos, de búsqueda de vivienda, trabajo, educación y socialización como una vía para no perder a aquellos pacientes que en base a una ayuda exclusivamente terapéutica nunca se vincularían a la red asistencial.

Los principios del "seguimiento asertivo" con los que se desarrolla el programa de intervención precoz son: 
INFORMES

-La intervención intensiva y móvil.

-Un servicio accesible las 24 horas del día los 7 días de la semana.

-Un contacto individual y único para cada usuario, cuidador o médico de cabecera a través de un key worker o trabajador clave identificado. (El rol y categoría profesional de dicho trabajador son explicados más abajo. Conservo el término inglés por no existir en nuestro medio sanitario público un rol similar con nombre en castellano).

-Continuidad: el servicio es flexible y continuado, adaptable a las necesidades cambiantes del paciente para evitar que éste tenga que hacer cambios de servicios o equipos.

-Se enfatiza el entorno comunitario aunque se reconoce la necesidad de hospitales en la misma medida que servicios basados en la comunidad.

Los criterios de admisión en el servicio son:

-edad entra 16 y 30 años.

-residencia en los distritos de referencia.

-diagnóstico de psicosis (alucinaciones, delirios, trastorno del pensamiento o alteración importante del comportamiento).

-no haber tenido contacto previo con servicios psiquiátricos.

Las vías de acceso al servicio son a través de:

-el servicio de interconsulta con atención primaria.

-los médicos de cabecera.

-equipos de urgencias psiquiátricas.

-otros como la derivación familiar, por la policía, asistencia social, etc.

Todos los casos nuevos son entrevistados por un equipo formado por un psicólogo clínico, un psiquiatra consultor y una enfermera mánager.

Una vez aceptado se le asigna a cada paciente un key worker que se encargará de facilitar la derivación desde el servicio de origen.

Todos los casos se siguen durante dos o tres años, o más en casos excepcionales, y al alta se derivan bien a atención primaria, al equipo de seguimiento asertivo o al de continuación de cuidados.

Papel y responsabilidad del «key-worker»

El "manejo" de la psicosis temprana es una actividad especializada que incluye:

- Una aproximación comprensiva, englobando en el concepto de recuperación perspectivas biológicas, psicológicas y familiares.

- El desarrollo de una fuerte alianza terapéutica.

- Habilidad para valorar las necesidades cambiantes del paciente y su familia.

- Habilidad para formular un plan de "manejo del caso". 
El key worker es una enfermera/o especializada en psiquiatría comunitaria que ha de reunir esas capacidades y además, en las distintas etapas de la enfermedad centrarse en las necesidades principales:

- Intervención en crisis y búsqueda de apoyos para la crisis.

- Reducción de síntomas y apoyo para minimizar riesgos.

- Ayuda para promover el proceso de recuperación.

- Ayudar al paciente a aceptar que tiene una vulnerabilidad para la psicosis y así promover la prevención de recaídas y la intervención precoz.

- Ayudar al paciente y su familia a comprender la enfermedad y sus consecuencias. social.

- Ayudar al paciente a alcanzar su máximo nivel de funcionamiento interpersonal y

Así, el éxito del programa de psicosis temprana depende de los key workers que son considerados la espina dorsal del servicio, alrededor de la cual se organizan los roles de los demás miembros del equipo (psiquiatras, psicólogos, terapeutas ocupacionales...).

Las habilidades clínicas del key worker deben incluir:

- Saber trasmitir al paciente la naturaleza de su enfermedad, especialmente la vulnerabilidad a las recaídas, sus probables consecuencias y los factores que están bajo su control.

- Proveer al paciente y sus cuidadores de información y promover un sentimiento de optimismo, control y autonomía.

- Apoyar al paciente y a su familia en las experiencias de la psicosis y así actuar como un ancla con la realidad que el paciente podrá usar cuando lo necesite.

- Desarrollar habilidades de afrontamiento para permitir al paciente tratar con los síntomas estresantes, e.g. voces...

Otros cometidos que puede desarrollar el key worker:

- Terapia cognitiva para síntomas resistentes a los tratamientos biológicos.

- Manejo de recaídas: reconocer la posibilidad de recaídas e identificación de los signos precoces de alarma con la puesta en marcha de un plan ensayado para desarrollar en su caso . Esto está basado en la llamada "firma de recaída": cada paciente posee una forma idiosincrática de comienzo de los episodios y si logramos identificar los síntomas que en cada uno de los pacientes los anuncian y que ellos mismos los reconozcan podrán actuar de forma rápida (según la intensidad de los síntomas un plan de actuación podría ser tomar más dosis de hipnótico si insomnio, confiarle sus preocupaciones a un familiar o amigo, telefonear a su key worker o acudir al centro a pedir ayuda) evitándose de esta forma el desarrollo del cuadro completo y los consiguientes riesgos.

- Intervención familiar: dos veces al año se organiza un curso de formación en terapia familiar de orientación cognitivo-conductual, al cual tuve la oportunidad de asistir, al que acuden aquellos key workers que desean llevar a cabo el programa de intervención familiar. 
En el caso de existir problemas difíciles o complejos de resolver el key worker puede hacer uso de otros profesionales solicitándolo a través de las reuniones de equipo semanales.

El key worker es el encargado de formular el plan de "manejo":

- Valorar las necesidades principales y factores de recaída.

- Desarrollar un plan para enganchar a tratamiento al paciente y establecer una alianza fuerte.

- Desarrollar una estrategia para situaciones de riesgo. de revisión.

- Hacer la presentación clínica del caso un mes tras la admisión y planear las fechas

\section{Actividades del programa de rehabilitación social}

El programa de rehabilitación social, que desarrollan dos terapeutas ocupacionales, está organizado en tres niveles de exigencia:

1) Los pacientes con dificultades graves de relación y aislamiento social requieren actividades de bajo nivel de exigencia para promover el enganche en el programa, como por ej. actividades lúdicas : natación, gimnasia, grupos exclusivamente de mujeres o de hombres que se reunen para mirar tiendas, ir a un pub o simplemente charlar.

2) En un nivel superior se sitúan los grupos de rehabilitación social en habilidades de la vida diaria llevados a cabo cuando es posible en la comunidad: grupos de cocina, entrenamiento en preparar los presupuestos de una vivienda, etc.

3) Por último, y en el nivel más exigente, están las actividades encaminadas a estructurar el rol y la red social del paciente, con ayuda para incorporar a los pacientes a cursos de formación profesional, o incluso búsqueda de empleo.

Se procura al máximo crear un entorno lo más agradable y atractivo para el paciente subrayándose la importancia de evitar el estigma. De este modo el lugar donde se atiende al paciente es en su casa, llevándose a cabo allí las entrevistas clínicas desde el principio: son los psiquiatras, los psicólogos y las enfermeras los que han de desplazarse al lugar donde se encuentra el paciente, no se espera que sea al revés, dada la dificultad con que, en muchas ocasiones, los sujetos aceptan el acudir a un centro psiquiátrico. Los clínicos se trasladan a visitar a los pacientes sin portar ningún distintivo externo (como batas blancas o coches tipo ambulancia) que pueda "asustar" o crear reticencias por parte del paciente. Cuando es necesario atender a pacientes en el centro, el key worker le va a buscar a su casa y le lleva al centro en su coche si hay la mínima dificultad de transporte público o de falta de iniciativa por parte del paciente.

Para las actividades de rehabilitación social se cuenta con un recinto en la planta baja del centro (una construcción de dos plantas con jardín), que es compartido con los 
INFORMES

pacientes que residen en la Unidad de Descanso y Recuperación, y que dispone de dos cocinas, una sala de televisión, y otra, la más concurrida, con una mesa de billar, equipo de música ... Allí tienen libre acceso todos los pacientes del centro y es su lugar de reunión: cualquiera puede llegar a cualquier hora del día, prepararse un té o un café, jugar una partida de billar o poner la música que quiera. Parece más un pub o club social que un centro psiquiátrico, y eso lo hace más atractivo para este tipo de pacientes, consiguiéndose así una buena vinculación al centro y a los terapeutas. Además, en las actividades sociales participa por igual personal y pacientes, organizándose viajes y excursiones planeadas conjuntamente y hasta un campeonato de fútbol quincenal, de staff contra pacientes, en el que juega incluso el director del servicio, el Prof. Birchwood.

\section{La unidad de descanso y recuperación}

La Unidad es abierta, tiene diez camas con una alta proporción profesionales/ usuarios: hay quince profesionales que cubren las 24 horas del día.

Cada paciente ingresado dispone de su propia habitación con toda la infraestructura, ninguna está decorada igual que otra (la apariencia es de un hostal, no la de un hospital), creando así un entorno de normalidad acorde con la filosofía general del centro de evitar el estigma. El principal objetivo es ofrecer un entorno hogareño y desinstitucionalizado, instando a los usuarios a tomar parte activa en el funcionamiento de la unidad.

El objetivo de esta unidad ( no es desde luego una planta de agudos, recurso que ya existe en el hospital de referencia) es ofrecer un servicio adecuado a las necesidades individuales de los pacientes que puede incluir cuidados de "reposo" (distanciando al paciente por ejemplo de una situación familiar estresante), apoyo intensivo en corta estancia o rehabilitación a medio plazo.

La derivación a esta unidad se hace desde el Servicio de Intervención Precoz. El usuario potencial es visitado en su casa o en el hospital por un miembro del personal de la unidad y otro del servicio. En esta entrevista se elabora un plan de cuidados basado en las necesidades del usuario y se valora el riesgo de suicidio. Antes de la admisión el usuario potencial visita la unidad y se le presenta al personal y a los compañeros y una vez admitido se le asigna un enfermero que coordinará todos los aspectos del cuidado del paciente durante su estancia. Éste firma un contrato en el que acepta seguir el programa de cuidados que ha sido elaborado por la enfermera nombrada, de forma que el usuario tome tanta responsabilidad en su tratamiento como sea posible. Dos psiquiatras consultores revisan el tratamiento médico mensualmente. Tras 4-6 semanas se lleva a cabo una reunión de revisión en la que han de estar el usuario, la enfermera nominada, el psiquiatra y cualquier otro miembro del equipo -si procede-, o familiar -si lo desea el paciente-. Allí se discute el progreso del usuario, sus cuidados a largo plazo y el plan de alta. Se espera que ningún usuario necesite más de 6 meses en la unidad. 
Para prevenir una crisis que podría terminar en hospitalización, la unidad ofrece estancias a corto plazo (máximo dos semanas) de "descanso", siendo necesaria la derivación por parte de un miembro del equipo del Servicio de Intervención Precoz.

\section{Conclusión}

Las experiencias de intercambio en la formación de los residentes, bajo mi punto de vista, y después de haber sido protagonista de una de ellas, deben fomentarse desde las instancias que organizan los planes de formación, ya que constituyen, no solo una forma de enriquecimiento profesional individual, sino también una vía para la entrada de nuevos y diferentes planeamientos para la práctica clínica y la organización de los servicios de salud. Hay que señalar que se trata de ideas ya puestas en práctica y de las que podemos incorporar los aspectos positivos sin obviar las dificultades que surgen a la hora de trasladar un modelo válido en un determinado contexto político y cultural a otro distinto.

El modelo inglés que he descrito es aplicable solo parcialmente al trabajo en salud mental comunitaria en España, pero da cuenta de una experiencia que ha demostrado ser útil sobre todo para mejorar la accesibilidad y la calidad de la atención a una parte de la población que habitualmente está en riesgo de quedar excluida de las redes de cuidados y que, sin embargo, constituye uno de los principales objetos de estudio de la psiquiatría de todos los tiempos y del campo de la salud mental actual: los pacientes psicóticos.

\section{Agradecimientos}

Este trabajo pudo llevarse a cabo gracias a la concesión de una Beca de Intercambio de la Comunidad de Madrid 
INFORMES

\section{BIBLIOGRAFÍA}

1- McGorry P.D., Edwards J., Mihalopoulos, Harrigan S.M., Jackson H.J, "EPPIC: An Evolving System of Early Detection and Optimal Management", Schizophrenia Bulletin, 22(2):305-326, 1996.

2- McGlashan T.H.,"Early Detection and Intervention in Schizophrenia: Research", Schizophrenia Bulletin, 22(2): 327-345, 1996.

3- Birchwood M, Todd P, Jackson C. "Early intervention in psychosis. The critical period hypothesis." British Journal of Psychiatry, 1998 , 172 (supl.33), 53-59.

4- Burnett R., Mallett R., Bhugra D., Hutchinson G., Der G., Leff J. "The first contact of patientes with Schizophrenia with psychiatric services: social factors and pathways to care in a multi-ethnic population", Psychological Medicine, 1999, 29, 475-483.

5- Birchwood M., Cochrane R., MacMillan F, Copestake S. KucharsKa J, Criss M., "The Influence of Ethnicity and family Structure on Relapse in First episode Schizophrenia. A comparison of Asian, afrocaribean and White Patients", British Journal of Psychiatry (1992), 161, 783-790.

* Médico interno residente de psiquiatría del Hospital General Universitario Gregorio Marañón de Madrid.

Correspondencia:

C/ Alcalde Sainz de Baranda 32, $3^{\circ}$ C , 28009 Madrid.

Fecha de recepción: 14-II-2000 